\title{
Automatic detection of microaneurysms using microstructure and wavelet methods
}

\author{
M TAMILARASI ${ }^{1, *}$ and K DURAISWAMY ${ }^{2}$ \\ ${ }^{1}$ Department of CSE, K.S.R College of Engineering, Tiruchengode 637215, India \\ ${ }^{2}$ Department of CSE, K.S. Rangasamy College of Technology, \\ Tiruchengode 637215, India \\ e-mail: replytotamil@yahoo.co.in; drkduraiswamy@yahoo.co.in
}

MS received 2 August 2013; revised 23 November 2014; accepted 25 December 2014

\begin{abstract}
Retinal microaneurysm is one of the earliest signs in diabetic retinopathy diagnosis. This paper has developed an approach to automate the detection of microaneurysms using wavelet-based Gaussian mixture model and microstructure texture feature extraction. First, the green channel of the colour retinal fundus image is extracted and pre-processed using various enhancement techniques such as bottom-hat filtering and gamma correction. Second, microstructures are extracted as Gaussian profiles in wavelet domain using the three-level generative model. Multiscale Gaussian kernels are obtained and histogram-based features are extracted from the best kernel. Using the Markov Chain Monte Carlo method, microaneurysms are classified using the optimal feature set. The proposed approach is experimented with DIARETDB0 and DIARETDB1 datasets using a classifier based on multilayer perceptron procedure. For DIARETDB0 dataset, the proposed algorithm obtains the results with a sensitivity of 98.32 and specificity of 97.59. In the case of DIARETDB1 dataset, the sensitivity and specificity of 98.91 and 97.65 have been achieved. The accuracies achieved by the proposed algorithm are 97.86 and 98.33 using DIARETDB0 and DIARETDB1 datasets respectively. Based on ground truth validation, good segmentation results are achieved when compared to existing algorithms such as local relative entropy-based thresholding, inverse adaptive surface thresholding, inverse segmentation method, and dark object segmentation.
\end{abstract}

Keywords. Retinal microaneurysms; wavelets; Gaussian mixture model; microstructure texture features; template matching.

\section{Introduction}

Diabetic retinopathy (DR) is the most common diabetic eye disease and a leading cause of blindness in the world. It can affect both eyes. People having DR often do not notice changes in their vision in the disease's early stages. But as it progresses, vision loss will occur. The cause of this

${ }^{*}$ For correspondence 
disease is due to changes in the blood vessels of the retina. Sometimes blood vessels swell and leak fluid or even close off completely. In other cases, abnormal new blood vessels grow on the surface of the retina.

In the world diabetes day celebrated on 14th November 2012, World Health Organization (WHO) reported that more than 346 million people worldwide have diabetes. According to WHO Prevention and Deafness Programme estimates reported by vision 2020 in world sight day 2012, about 285 million people are visually impaired worldwide: out of which 39 million are blind and 246 million have low vision. India has 31.7 million diabetic subjects, and the number is expected to increase to a staggering 79.4 million by 2030 .

The best treatment for DR is to prevent it. Treatment usually will not cure DR but it may slow down the progression of vision loss. Without treatment, DR progresses steadily from minimal to severe stages. Earlier diagnosis can delay the progression of this disease and consequent visual loss.

Recognition of microaneurysms is the most challenging task for reliable automatic detection and diagnosis of diabetic retinopathy. A number of studies have been investigated on diabetic retinopathy. This paper deals with the recently introduced methods for analyzing the microaneurysms detection, available for automated diagnosis. Although many algorithms have been developed, the accurate detection of microaneurysm in colour retinal images is still a challenging problem. Automating this task, which is currently performed manually, would bring more objectivity and reproducibility. This helps ophthalmologist to diagnose the disease early and determine the treatment for the patient.

Many of the studies investigated that there are different types of algorithms for automatic detection of microaneurysms in fluorescein angiography retinal fundus images. Meysam Tavakoli et al (2013) presented a novel algorithm based on radon transform and multioverlapping windows for the automatic detection of microaneurysms in fundus images Top-hat transformation and averaging filter techniques are applied to remove the background for preprocessing. After pre-processing, the whole image is divided into sub-images. Optic nerve head $(\mathrm{ONH})$ and vessel tree are then detected and masked by applying radon transform in each subimage. After detecting and masking retinal vessels and $\mathrm{ONH}$, microaneurysms are detected and numbered using radon transform and thresholding. The proposed method is evaluated on three different retinal images databases such as the mashhad database with 120 fundus images, second local database from Tehran with 50 retinal images and retinopathy online challenge database with 22 images. Results have achieved a sensitivity and specificity of $94 \%$ and $75 \%$ for Mashhad database and $100 \%$ and $70 \%$ for the second local database respectively.

Marwan D Saleh \& Eswaran (2012) provides an automated decision-support system for nonproliferative diabetic retinopathy disease based on microaneurysms and haemorrhages detection Some foreground objects, such as optic disk, fovea, and blood vessels, were extracted for accurate segmentation of dark spot lesions in the fundus images. Dark object segmentation approach was used to locate abnormal regions such as microaneurysms and haemorrhages. Based on the number and location of microaneurysms and haemorrhages, the system evaluates the severity level of DR. In the proposed system, dark spot lesions such as microaneurysms and haemorrhages were segmented using step-by-step approach such as h-maxima transformation, thresholding and feature extraction. The pre-processed image was further processed by the hmaxima transform for suppressing all maxima in the intensity image whose values were less than a certain threshold. In this stage, both microaneurysms and haemorrhages were segmented by thresholding the intensity image. In the proposed algorithm, multilevel thresholding technique was used to produce the binary image. Based on seven features, different spot lesions such as cotton-wool spots, hard exudates, drusen, microaneurysms, and haemorrhages can be 
classified. Features considered are size, shape, roughness, edge sharpness, brightness, colour, and depth. Microaneurysms and haemorrhages are similar with respect to five features, which are dull (roughness), insignificant edge (edge sharpness), dark (brightness), reddish (colour), and superficial (depth). Hence the classification was mainly based on the remaining two features, i.e. size and shape. Overall, the proposed system yields sensitivity and values of $89.47 \%$ and $95.65 \%$ for the classification of DR versus normal.

Fangyan et al (2013) proposed a novel two-dimensional variance thresholding scheme to improve image segmentation. One drawback of $1 \mathrm{D}$ thresholding methods is that only the distribution of the gray levels of an image is considered, whereas the spatial information is ignored. To overcome this problem, 2D variance-based techniques using spatial as well as pixel information have been proposed. This scheme uses 1D summation. It is almost as fast as the original 1D variance-based algorithm. In this scheme, the gray levels of the pixels and the local average gray level of the neighbourhood pixels form a 2D histogram. This 2D parameter space is reduced to a 1D histogram, while assigning equal weights to both variables. Experimental results on bi-level and multilevel thresholding for synthetic and real-world images demonstrate that the proposed image thresholding scheme performs well compared with the Otsu method, 2-d Otsu method and the minimum class variance thresholding method.

Usman Akram M \& Shoab A Khan (2013) proposed a three-stage system for early detection of microaneurysms using filter banks. All possible candidate regions for microaneurysms that present in retinal image were extracted by the proposed system. A feature vector for each region depending upon certain properties, i.e. shape, colour, intensity and statistics was formed to classify a candidate region as microaneurysms or non- microaneurysms. A hybrid classifier which combines the Gaussian mixture model, support vector machine and an extension of multi-model mediod based modelling approach in an ensemble was presented to improve the accuracy of classification. The true microaneurysms regions were selected and classified using a hybrid classifier which was a weighted combination of multivariate m-Mediods, Gaussian mixture model and support vector machine. The proposed system had achieved higher accuracy which was better than previously published methods.

Istvan Lazar \& Andras Hajdu (2013) proposed a method for retinal microaneurysms detection through local rotating cross-section profile analysis. Microaneurysms detection was recognized by this approach through the analysis of directional cross-section profiles centred on the local maximum pixels of the pre-processed image. Peak detection was applied on each profile, and a set of attributes regarding the size, height, and shape of the peak are calculated subsequently. Attribute values were used as the orientation of the cross-section changes. These values constitute the feature set that was used in a Naive Bayes classification to eliminate false candidates. The final score of the remaining candidates can be thresholded further for a binary output. The proposed method was tested with the retinopathy online-challenge and proved to be competitive with the existing approaches. The proposed method had achieved a higher sensitivity at low false positive rates, i.e., at 1/8 and 1/4 false positives/image.

Bob et al (2012) presented a new method to detect microaneurysms and retinal blood vessels based on dictionary learning with sparse representation classifier. This method consists of two phases. First, all possible microaneurysms candidates were identified with the help of multi-scale Gaussian correlation filtering. The second step was to classify these candidates with dictionary learning via sparse representation classifier. Two dictionaries were used in this proposed approach: one for the microaneurysms and other for the non-microaneurysms. Vessel extraction was based on multi-scale production of matched filter and sparse representation classifier. First, vessel centre-line candidates are extracted using multi-scale matched filtering, scale production, double thresholding and centre-line detection. Then, the candidates which are centre-line pixels 
were classified. Two dictionary elements of vessel and non-vessel were used in the sparse representation classifier process. Experimental results showed that the proposed method can well distinguish microaneurysms from non- microaneurysms objects.

Cemal et al (2012) developed an approach called inverse segmentation method to detect DR. Direct segmentation techniques give poor results in some of the cases. The proposed system exploits the homogeneity of healthy areas rather than dealing with varying structure of unhealthy areas for segmenting bright lesions (hard exudates and cotton wool spots). This system first generates the reference or extended background image from a retinal image. Healthy parts of the retinal image except for vessel and optic disk areas were used in the calculation of this reference image. Next the retinal image was divided into two parts as low and high intensity areas based on the intensities of the background image. Background image was used as the dynamic threshold value for segmenting high intensity and low intensity degenerations in the image. Both degenerations were segmented separately by using the inverse segmentation method and dynamic thresholding. Due to the use of dynamic threshold, the proposed method adapts itself to intensity changes across the whole image and achieves good segmentation results. To compare with existing methods, a Naïve Bayes method was applied for segmentation of DR. The performance of the system was over $95 \%$ in detection of the optic disk, and $90 \%$ in segmentation of the DR. Therefore, the method provides high segmentation and measurement accuracy. In some cases, the image lighting artifacts may affect segmentation performance negatively, which could also be considered as an issue.

A two-phase decision support framework (Balint et al 2012) is proposed for the automatic screening of digital fundus images. Pre-screening is the first step in which images are classified as severely diseased (highly abnormal) or to be forwarded for further processing. The second step of the proposed method detects regions of interest with possible lesions on the images that previously passed the pre-screening step. These regions will serve as input to the specific lesion detectors for detailed analysis. The computational performance of a screening system is increased due to pre-screening process. Experimental results show that there is a decrease in the computational burden of the automatic screening system.

An Ensemble-Based System (Balint \& Andras 2012) is developed for Microaneurysm Detection and Diabetic Retinopathy Grading. This approach has proved its high efficiency in an open online challenge with its first position. Our novel framework relies on a set of $<$ pre-processing method, candidate extractor $>$ pairs. A search algorithm is used to select an optimal combination. Since the proposed approach is modular, further improvements can be done by adding more preprocessing methods and candidate extractors. The DR/non-DR grading performance of this detector in the 1200 images of the Messidor database have achieved a $0.90 \pm 0.01$ area under receiver operating characteristic curve, which is competitive with other existing methods.

Anderson et al (2012) presented a common approach for identifying both red and bright lesions in DR images without requiring specific pre- or post-processing. The proposed approach requires pinpointing the location of each lesion to allow the specialist to evaluate the image for diagnosis. It constructs a visual word dictionary representing points of interest located within regions marked by specialists. Fundus images are classified as normal or DR-related pathology based on the presence or absence of these points of interest. The area under the curve of $95.3 \%$ and $93.3 \%$ is achieved for white and red lesion detection using fivefold cross validation. The visual dictionary is robust for DR screening of large, diverse communities with varying cameras and settings and levels of expertise for image capture.

A new approach based on multi-scale correlation filtering (MSCF) and dynamic thresholding is developed for detecting microaneurysms using multi-scale correlation coefficients (Bob et al 2010). There are two levels, microaneurysm candidate detection (coarse level) and true 
microaneurysm classification (fine level). The proposed approach is evaluated using two public datasets-Retinopathy on-line challenge, and DIARETDB1 (standard diabetic retinopathy database). The task for coarse-level detection identifies all possible microaneurysm candidates in a retinal image. It applies a sliding neighbourhood filter with multi-scale Gaussian kernels to the fundus image to calculate a correlation coefficient for each pixel. Microaneurysms are circular in shape. Gaussian kernels are also circular. The response of the filtering will be high for the candidate region. Five scales are used for the kernel to represent microaneurysms of different sizes with the sigma of the Gaussian function being 1.1, 1.2, 1.3, 1.4 and 1.5. The final response is obtained by combining the maximum coefficients from each of the five responses. In the fine level detection, true microaneurysms are classified using 31 features extracted from the level one candidates.

Carla et al (2010) have proposed the use of multiscale amplitude-modulation-frequencymodulation methods for discriminating between normal and pathological retinal images. The proposed method was tested using standard images from the early treatment diabetic retinopathy study. The different types of pathologies considered in this work were microaneurysms, exudates, and neovascularization on the retina, haemorrhages, normal retinal background, and normal vessels patterns. The proposed system aims to characterize the normal and pathological retinal structures based on cumulative distribution functions of the instantaneous amplitude, the instantaneous frequency magnitude, and the relative instantaneous frequency angle from multiple scales. These functions were used as texture feature vectors. Inter-structure similarity was measured using the distance between the extracted feature vectors. Experimental results demonstrated the difference between normal retinal structures and pathological lesions based on amplitude-modulation-frequency-modulation features.

Alan D Fleming et al (2006) described an automatic method for microaneurysms detection using local contrast normalization and local vessel detection. This approach improves the ability to distinguish between microaneurysms and other dots that occur on the retina. Variety of methods for contrast normalization were compared. Watershed retinal region growing method was used to derive a region that contains no vessels or other lesions. Dots within vessels were handled successfully using a local vessel detection technique. Experimental results achieved a sensitivity $85.4 \%$ and specificity $83.1 \%$ for detection of individual microaneurysms and for detection of images containing microaneurysms.

Giri Babu Kande et al (2010) have proposed a novel method for red lesion detection in fundus images based on pixel classification and mathematical morphology. The proposed algorithm uses the intensity information from red and green channels of the same retinal image and the thresholding based on local relative entropy. For efficient detection of red lesions, it is desirable to have high contrast between red lesions and the retinal background while there should be low contrast between the retinal background and bright lesions. Combining the advantages of both channels, brightness in red channel and high contrast in green channel, results in increasing the contrast between red lesions and retinal background and decreasing the contrast between bright lesions and the retinal background. This results in the effective reduction of false positives during candidate red lesion extraction. Local entropy thresholding algorithm, which takes into account the spatial distribution of gray levels, performs efficiently in distinguishing between enhanced red lesion segments and the background since it can preserve the structure details of an image. The proposed method performs very well in detecting red lesions even in low-contrast regions as the intensity information of both red and green channels is used.

Thomas et al (2007) proposed an algorithm for automatic detection of microaneurysms in colour fundus images. There are four steps in the proposed algorithm. The first step consists of image enhancement, shade correction and image normalization of the green channel. The second 
step was candidate detection which detects patterns corresponding to microaneurysms using diameter closing and an automatic threshold scheme. The third step was the feature extraction. The extracted features were used in the last step to automatically classify candidates into real microaneurysms and other objects. The last step was the classification which relies on kernel density estimation with variable bandwidth. The proposed algorithm was experimented with a database of 21 annotated images. A sensitivity of $88.5 \%$ was achieved compared with manually obtained gradings of 94 images.

Meindert et al (2005) have developed an automated method to detect red lesions with two important contributions. The first contribution is a new red lesion candidate detection system based on pixel classification. Second, an extensive number of new features are added to those proposed by Spencer-Frame. The detected candidate objects are classified using all features and a k-nearest neighbour classifier with a very high sensitivity and a reasonable specificity. In the first stage, each image is pre-processed. Candidate objects that represent red-lesions are extracted in the second stage. In the final stage, the probability for each candidate to represent a red-lesion is estimated using a classifier and a large set of specifically designed features. The performance of this scheme is compared to the detection scheme based on mathematical morphology used by Spencer-Frame and also to the hybrid scheme that combines both detection strategies. To improve the classification of the candidate objects into lesion and non-lesion, an extensive number of features have been added to the set proposed by Spencer-Frame. Results have been tested with several classifiers. Best results are obtained with k-nearest-neighbours classification.

This paper has developed the wavelet-based template matching algorithm to detect microaneurysms. Gaussian mixture model and microstructure feature extraction are used for the segmentation of abnormal retinal lesions. In this paper, wavelets are used to automate the multiscale Gaussian kernels for profile construction. A wavelet domain template learning algorithm uses a three-level generative model for learning templates from images. From this model, the base and texton maps are constructed. Textons are modelled as Gaussian mixtures using texture laws. Histogram-based texture features are extracted from the best Gaussian function and used for classifying red lesion such as microaneurysms. The proposed algorithm has achieved better segmentation results than the conventional segmentation algorithms.

This paper consists of four sections. Section 2 consists of the concepts used in the proposed algorithm. Section 3 consists of results and discussion and section 4 is the conclusion.

\section{Materials and methods}

The overall architecture of the proposed system is depicted in figure 1 .

\subsection{Image pre-processing}

Colour fundus images often suffer from non-uniform illumination, poor contrast and noise. The green channel of the RGB-representation shows the best contrast. The red channel is often saturated and has low contrast. The blue channel is very noisy and suffers from poor dynamic range. This does not mean that there cannot be any useful information in the red and blue channels. To improve the accuracy of abnormal signs of DR, the green channel of the image is enhanced using pre-processing techniques such as bottom hat filtering and gamma correction. Bottom-hat transformation provides a functionality for subtracting original image from a morphologically closed version of a binary or gray-scale image. To control the overall brightness of an image, gamma correction technique is applied. The gamma value is automatically computed based on 


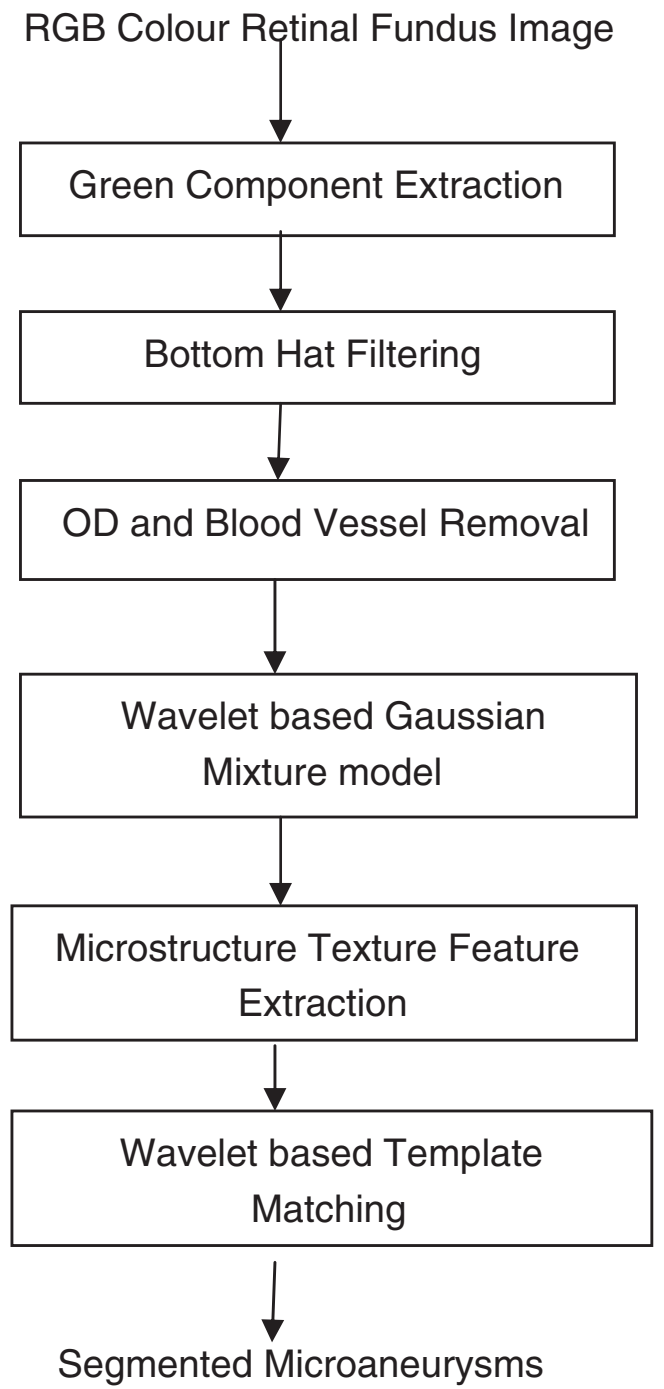

Figure 1. Proposed system architecture.

the cumulative histogram of an image. A proper estimation of gamma value enhances the contrast of the image. For further improvement, anatomical structures such as optic disk and blood vessel structures are detected and removed.

\subsection{Optic disk removal}

If objects of interest have other shapes than lines, it could be parables, circles or ellipses or any other arbitrary shape. The general Hough transform can be used on any kind of shape, although the complexity of the transformation increases with the number of parameters needed to describe the shape. In this paper, Circular Hough Transform (CHT) is used to extract the optic disk in retinal fundus images. CHT can be described as a transformation of a point in the $x, y$-plane 
to the parameter space. The parameter space is defined according to the shape of the object of interest. The circle is actually simpler to represent in parameter space, compared to the line, since the parameters of the circle can be directly transferred to the parameter space. The equation of a circle is defined as

$$
r=(x-a)^{2}+(y-b)^{2}
$$

The circle got three parameters, $r, a$, and $b$, where $a$ and $b$ are the centre of the circle in the $x$ and $y$ direction respectively and $r$ is the radius. The parametric representation of the circle is defined as follows:

$$
\begin{aligned}
& x=a+r \cos (\theta) \\
& y=b+r \sin (\theta)
\end{aligned}
$$

In order to simplify the parametric representation of the circle, the radius can be held as a constant or limited to number of known radii. To improve the process of finding circle in an image using CHT, edges are detected in the image using morphological operation. At each edge point, a circle is drawn with centre in the point with the desired radius. This circle is drawn in the parameter space, such that the $x$ axis is the $a$-value and the $y$ axis is the b-value while the $z$ axis is the radii. At coordinates, which belong to the perimeter of the drawn circle, the value is incremented in the accumulator matrix, which has the same size as the parameter space. For every edge point in the input image, circle is drawn with the desired radii and accumulator values are incremented. The accumulator contains numbers corresponding to the number of circles passing through the individual coordinates. Thus the highest number corresponds to the centre of the circles in the image. Multiple circles with the same radius can be found with the same technique. Overlap of circles can cause spurious centres to be found. Spurious circles can be removed by matching to circles in the original image. Figure 2 shows the results of $\mathrm{CHT}$ with different edge detection techniques such as sobel, canny, prewitt, zero crossing and morphologic approach.

\subsection{Blood vessels removal}

The matched filter is one of the template matching algorithms that is used in the detection of the blood vessels in retinal images and other application as well. It is based on the spatial properties of the object to be recognized. The idea of the matched filter is introduced by taking a number of samples for a cross section of retinal blood vessels as stated by Mohammed et al (2007). The gray level profile of these samples is then approximated by a Gaussian-shaped curve. Matched filter design is based on the number of properties for blood vessels. The properties are given as follows

- Vessels can be approximated as anti-parallel segments.

- Vessels have lower reflectance than other retinal surfaces. They appear darker relative to the background.

- Vessel size may decrease when moving away from the optic disk, the width of a retina vessel may lie within the range of $2-10$ pixels.

- The intensity profile varies by a small amount from vessel to vessel.

- The intensity profile has a Gaussian shape.

To be able to detect vessels on all possible orientations, the kernel must be rotated to all possible vessel orientations and the maximum response from the filter bank is registered. The output of matched filter is a continuous image. Finally, thresholding procedure is used to segment the blood vessels. Using this method, the average accuracy of the segmented images is 0.97 


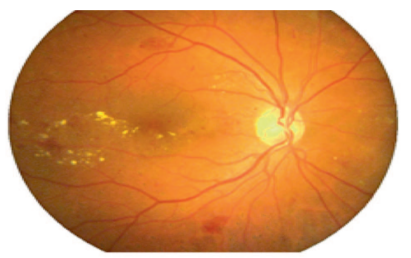

(a)

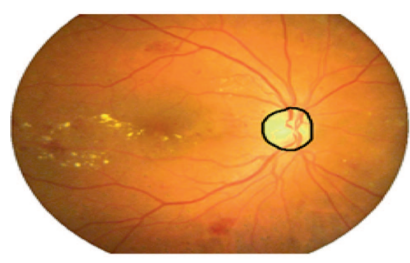

(c)

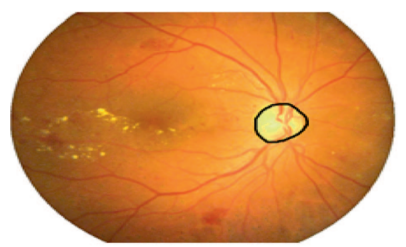

(e)

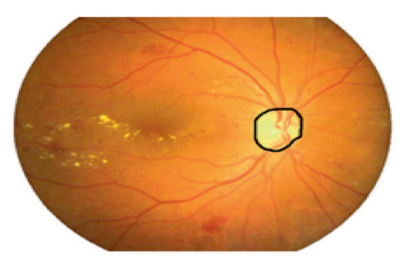

(b)

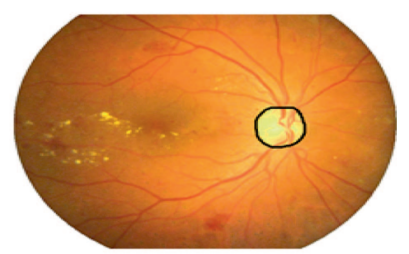

(d)

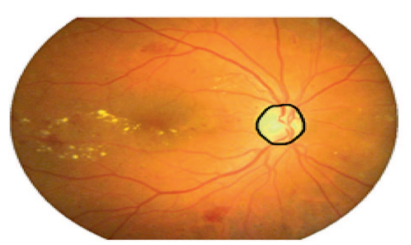

(f)

Figure 2. Optic disk detection: (a) Original image, (b) Sobel-based CHT, (c) Canny-based CHT, (d) Prewitt-based CHT, (e) Zero crossing-based CHT, (f) Morphologic-based CHT.

compared to the maximum average accuracy which is 0.98 . Figure 3 shows the original image and the blood vessel detected image using matched filter.

\subsection{Wavelet-based Gaussian mixture model}

Wavelets have been used with great success in applications, such as signal denoising, compression, estimation and feature extraction, because of their ability to capture the singularities in the signal with a few coefficients. Wavelet domain statistical image models and hierarchical pattern models are fused in this paper. A deformable template was learnt from a set of training observations. The local deformations are modelled in the wavelet domain by treating the template to be statistical in nature. These are modelled by treating the wavelet coefficients as random variables. The global deformations are modelled as spatial transformations that exhibit a welldefined adjoint. The existence of well-defined adjoint provides the flexibility of deforming either the template or the pattern itself.

The Gaussian Mixture Model (GMM) is used for modelling wavelet coefficients but the form that is generally used assumes two zero mean Gaussians, one with a low variance and the other with a high variance. For template learning, different forms of GMM are used because the local statistics of wavelet coefficients need to be taken into consideration. The pattern and background information need to be modelled efficiently, so that they can be clearly distinguished. The GMM proposed is a two-state model with a zero mean and a non-zero mean. The state Qi $=1$ represents a zero mean Gaussian and the state $\mathrm{Qi}=2$ represents the Gaussian with non-zero mean. Each wavelet coefficient is assumed to have $\mu_{i, 2}$ and $\sigma_{i, 2}^{2}$ whereas $\mu_{i, 1}=0$ and $\sigma_{i, 1}^{2}$ is constrained to 

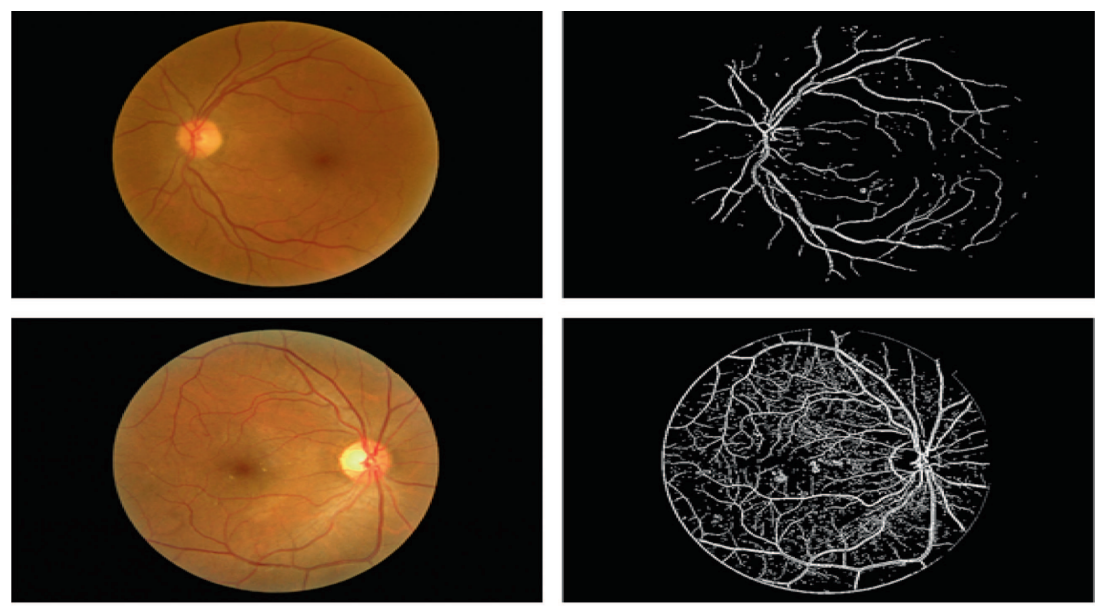

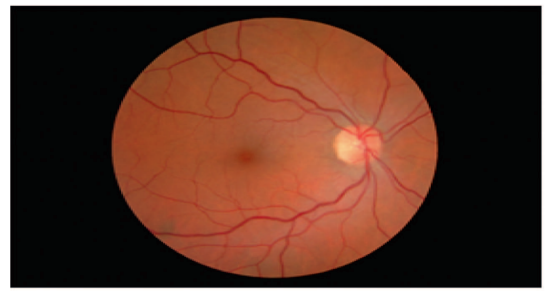

(a)

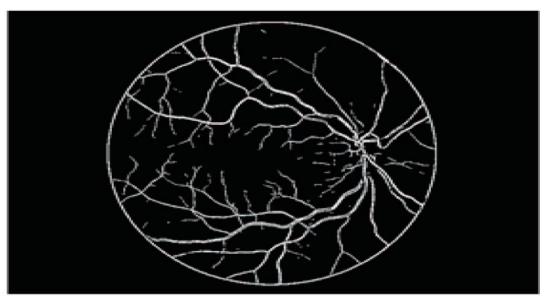

(b)

Figure 3. Matched filter (a) Original image, (b) Blood vessel detected image.

be same for all the coefficients in a subband. In this case, the Gaussian corresponding to Q $i=0$ has a high variance and zero mean whereas, the Gaussian corresponding to Qi $=1$ has a lower variance and a mean of 3 . The state $\mathrm{Qi}=0$ models the background and smooth regions with a zero mean and common variance across the subband, whereas the state $\mathrm{Q} i=1$ corresponds to the pattern. For convenience, Qi $=1$ is referred to as the low state for the coefficient $\mathrm{i}$ and Qi $=2$ is referred to as the high state. This model also agrees with the intuition that less parameters must be used to model the background and more parameters must be used for the actual pattern itself.

\subsection{Microstructure texture feature extraction}

Texture analysis characterizes the texture through first or second order statistics, through a model, through the spatial relations between pixels or through a transform (Fourier, Gabor, and Wavelet). Images are analyzed from the point of view of its specific microstructures: ridges, edges, spots, waves, ripples, grooves. Laws convolution filters are used in order to detect these microstructures. The Laplacian of Gaussian ( $\mathrm{LoG})$ is used to obtain the intensity profile. Frequency of microstructures is computed as

$$
\begin{aligned}
& \text { ridges - rough surfaces } \\
& \text { spots - pitches } \\
& \text { edges - cracks }
\end{aligned}
$$

The texton-based method is invariant to changes in orientation and illumination. Textons correspond to the microstructures in the texture. Texture features are extracted using the Laws 
convolution filters, and the Laplacian of Gaussian. Laws convolution filters are Level, Edge, Spot, Wave and Ripple. Texton formations group the feature vectors into classes using the k-means clustering method. The steps in the texton-based method are

- Find textons in the given image and mark each pixel with the corresponding texton label

- Split the image into small enough blocks and compute the textons histogram for each block

- Compare the histograms of the neighbouring blocks, in the horizontal direction (chi-square distance)

$$
\chi^{2}\left(h_{1}, h_{2}\right)=\frac{1}{2} \sum_{n=1}^{\# \text { bins }} \frac{\left(h_{1}(n)-h_{2}(n)\right)^{2}}{h_{1}(n)+h_{2}(n)}
$$

- Decide a texture border if the chi-squared distance between the histograms overpasses the threshold:

$$
\text { Threshold }=\frac{\chi_{\min }^{2}+\chi_{\max }^{2}}{2}+\sigma_{\chi}^{2}
$$

where $\chi_{\min }^{2}$ and $\chi_{\max }^{2}$ represent the minimum and maximum values of the distances computed, from left to right, between the neighbouring blocks of the image, $\sigma_{\chi}^{2}$ is the squared variance of these distances.

- Compare the textons histogram with some histograms previously stored in the training set, corresponding to the texture of the microaneurysms.

Other features like size and shape are used in order to distinguish the microaneurysms from other lesions. Law's texture features observed that certain gradient operators such as Laplacian and Sobel operators accentuated the underlying microstructure of texture within an image. This is the basis for a feature extraction scheme based a series of pixel impulse response arrays obtained from combinations of 1-D vectors. Each 1-D array was associated with an underlying microstructure and labelled using an acronym accordingly. The arrays are convolved with other arrays in a combinatorial manner to generate a total of 25 masks, typically labelled as L5L5 for the mask resulting from the convolution of the two L5 arrays. Five 1-D arrays identified by Laws are

Level L5 $=\left[\begin{array}{lllll}1 & 4 & 6 & 4 & 1\end{array}\right]$

Edge $\mathrm{E} 5=\left[\begin{array}{lllll}-1 & -2 & 0 & 2 & 1\end{array}\right]$

Spot S5 $=\left[\begin{array}{lllll}-1 & 0 & 2 & 0 & -1\end{array}\right]$

Wave W5 $=\left[\begin{array}{lllll}-1 & 2 & 0 & -2 & 1\end{array}\right]$

Ripple R5 = $\left[\begin{array}{lllll}1 & -4 & 6 & -4 & 1\end{array}\right]$

These masks are subsequently convolved with a texture field to accentuate its microstructure. The energy of the microstructure arrays is measured together with other statistics. Five computed amplitude features are mean, standard deviation, skewness, kurtosis, and energy measurements. Since there are 25 different convolutions, altogether a total of 125 features are obtained. Waveletbased Gaussian mixture model is used to eliminate any features that are weak in their ability to differentiate between different classes. The final set of features is used for all further analysis. Texture transformation is applied to an image using texture laws. This is done by convolving the input image with a special filter mask. The filters used are:

9 different $3 \times 3$ matrices obtainable from the following three vectors:

$$
1=\left[\begin{array}{lll}
1 & 2 & 1
\end{array}\right],
$$




$$
\begin{aligned}
& \mathrm{e}=\left[\begin{array}{lll}
-1 & 0 & 1
\end{array}\right], \\
& \mathrm{s}=\left[\begin{array}{lll}
-1 & 2 & -1
\end{array}\right]
\end{aligned}
$$

25 different $5 \times 5$ matrices obtainable from the following five vectors:

$$
\begin{aligned}
& 1=\left[\begin{array}{lllll}
1 & 4 & 6 & 4 & 1
\end{array}\right], \\
& \mathrm{e}=\left[\begin{array}{lllll}
-1 & -2 & 0 & 2 & 1
\end{array}\right], \\
& \mathrm{s}=\left[\begin{array}{lllll}
-1 & 0 & 2 & 0 & -1
\end{array}\right], \\
& \mathrm{w}=\left[\begin{array}{lllll}
-1 & 2 & 0 & -2 & 1
\end{array}\right], \\
& \mathrm{r}=\left[\begin{array}{lllll}
1 & -4 & 6 & -4 & 1
\end{array}\right]
\end{aligned}
$$

49 different $7 \times 7$ matrices obtainable from the following seven vectors:

$$
\begin{aligned}
& \mathrm{l}=\left[\begin{array}{lllllll}
1 & 6 & 15 & 20 & 15 & 6 & 1
\end{array}\right], \\
& \mathrm{e}=\left[\begin{array}{lllllll}
-1 & -4 & -5 & 0 & 5 & 4 & 1
\end{array}\right], \\
& \mathrm{s}=\left[\begin{array}{lllllll}
-1 & -2 & 1 & 4 & 1 & -2 & -1
\end{array}\right], \\
& \mathrm{w}=\left[\begin{array}{lllllll}
-1 & 0 & 3 & 0 & -3 & 0 & 1
\end{array}\right], \\
& \mathrm{r}=\left[\begin{array}{lllllll}
1 & -2 & -1 & 4 & -1 & -2 & 1
\end{array}\right], \\
& \mathrm{u}=\left[\begin{array}{llllllll}
1 & -4 & 5 & 0 & -5 & 4 & -1
\end{array}\right], \\
& \mathrm{o}=\left[\begin{array}{lllllll}
-1 & 6 & -15 & 20 & -15 & 6 & -1
\end{array}\right]
\end{aligned}
$$

The names of the filters are mnemonics for "level," "edge," "spot," "wave," "ripple," "undulation," and "oscillation." For most of the filters, the resulting gray values must be modified by a shift. This makes the different textures in the output image more comparable to each other. The name of the filter is composed of the letters of the two vectors used, where the first letter denotes convolution in the column direction while the second letter denotes convolution in the row direction. The default size of the filter kernel used is 5. The default value used is 'el'. The suggested values are defined as the following:

$$
\begin{aligned}
& \text { 'll', 'le', 'ls', 'lw', 'lr', 'lu', 'lo', ‘el', 'ee', 'es', ‘ew', 'er', 'eu', 'eo', 'sl', 'se', 'ss', } \\
& \text { 'sw', 'sr', ‘su', 'so', 'wl', 'we', 'ws', 'ww', 'wr', 'wu', 'wo', 'rl', 're', 'rs', 'rw', } \\
& \text { 'rr', 'ru', 'ro', ‘ul', 'ue', 'us', ‘uw', ‘ur', ‘uu', ‘uo', 'ol', 'oe', 'os', ‘ow', ‘or', ‘ou', } \\
& \text { 'oo' }
\end{aligned}
$$

\subsection{Template matching in wavelet domain}

Template matching in the temporal and spatial domain is one of the earliest known methods for pattern classification. Pattern classification has grown as one of the key areas of research that finds major applications in the present day automated systems. A wavelet domain template learning algorithm uses a three-level generative model for learning templates from images. An image $\mathrm{I}$ is a superposition of a number of image bases selected from an over-complete dictionary $\Psi$ including various Gabor and Laplacian of Gaussian bases at various locations, scales, and orientations. The image bases are represented as a base map B. The base map B is, in turn, generated by a smaller number of texton elements, denoted by a texton map T. Textons considered for this work include geometric structures and photometric structures. For geometric structures, a texton consists of a small number of image bases with deformable spatial configurations. The geometric structures are learned from a static texture image with repeated elements. For photometric structures, a texton represents a three-dimensional surface element under varying illuminations and is called a lighton. A lighton is a triplet of $2 \mathrm{D}$ textons. For a given light source, a lighton image is generated as a linear sum of the three textons. The texton elements are selected from 
a dictionary of textons $\Pi$. The base map B and texton map $\mathrm{T}$ are hidden variables and the dictionaries $\Psi$ and $\Pi$ are parameters that should be learned through fitting the model to observed images. Textons are represented as Gaussian mixtures. The final feature vector is obtained from the feature values of the highest Gaussian function. Red lesions can be segmented and classified using the final feature set.

Markov Chain Monte Carlo refers to a class of algorithms for sampling from probability distributions in a special way. It does this by constructing a Markov chain which converges after a certain number of steps to the desired probability distribution. A Markov chain is a process in which the next step or iteration of the process only depends upon the current step and not upon any previous steps in the process. If a Markov Chain has certain properties then the process will evolve in a random fashion until it reaches a certain state in which it remains thereafter, called equilibrium. Within the context of Bayesian statistics it is desirable to sample from a posterior distribution. An MCMC algorithm constructs a Markov chain in which the random evolution of the chain is probabilistic, each step in the chain constructs an empirical distribution which is a Monte Carlo approximation of the posterior, and the chain converges to an equilibrium distribution which is the posterior distribution.

The proposed wavelet domain template learning algorithm consists of two stages namely, training and testing. The steps are given below

\section{Training}

- Apply three-level generative model for a number of images taken under different orientation and illumination conditions.

- Construct base map a number of image bases selected from an over-complete dictionary $\Psi$ including various Gabor and Laplacian of Gaussian bases at various locations, scales, and orientations.

- Create different textons from the different bases in base map to form the texton map.

- Initialize the hidden Markov chain with texton map using the three-level generative model.

- Obtain texton maps using different scales and orientations for the next level.

- Update texton templates if there is an improvement in minimum description length. Otherwise stop the chain.

- Apply texture laws to each texton and model the output as Gaussian mixtures.

- Select the Gaussian function with highest feature values.

- Obtain the feature vector for the red lesion from the feature values of the selected Gaussian function.

\section{Testing}

- Use a single image, under arbitrary orientation and illumination conditions.

- Use Markov-chain-Monte-Carlo method in order to decide the most probable configuration of textons and the most probable class.

\section{Algorithm: Markov-Chain-Monte-Carlo Method}

Repeat

Randomly assign to each pixel in the image the label of a texton, to which it probably corresponds.

Compute the probabilities of belonging to the classes.

Until convergence 


\section{Results}

The proposed segmentation algorithm is experimented using DIARETDB0 and DIARETDB1datasets. DIARETDB0 consists of 130 colour fundus images of which 20 are normal and 110 contain signs of the diabetic retinopathy (hard exudates, soft exudates, microaneurysms, haemorrhages, and neovascularization). Images are captured with a 50-degree field-of-view digital fundus camera with unknown camera settings. The data can be used to evaluate the general performance of diagnosis methods. This dataset is referred to as calibration level 0 fundus images. DIARETDB1 consists of 89 colour fundus images. Eighty four images contain at least mild non-proliferative DR signs (microaneurysms) and five are considered normal, not containing DR signs according to all experts who participated in the evaluation. Images are captured using the same 50-degree field-of-view digital fundus camera with varying imaging settings. Four medical experts are asked to mark the areas related to the microaneurysms, haemorrhages, and hard and soft exudates. Ground truth confidence levels, $\{<50 \%, \sim>50 \%$, $\sim 100 \%\}$, represented the certainty of the decision that a marked finding is correct, are included.

Compared with the existing algorithms such as local relative entropy-based thresholding, inverse adaptive surface thresholding, inverse segmentation method, and dark object segmentation, the proposed algorithm achieves good segmentation result which is shown in figure 4 . The green channel of the image is extracted and pre-processed using various contrast enhancement techniques. Profile is constructed using Gaussian mixture in wavelet domain. From this profile, intensity changes are analysed and segmented the microaneurysms using wavelet-based template

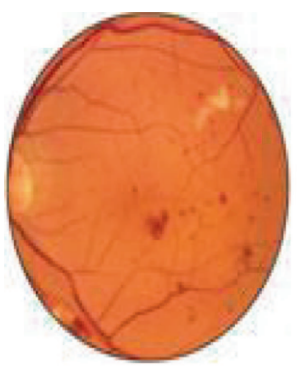

(a)

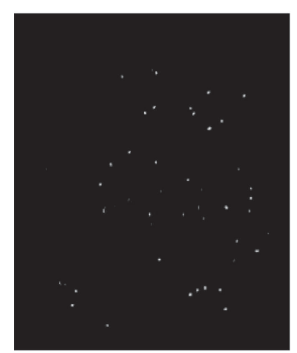

(e)

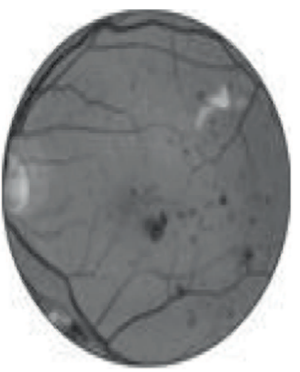

(b)

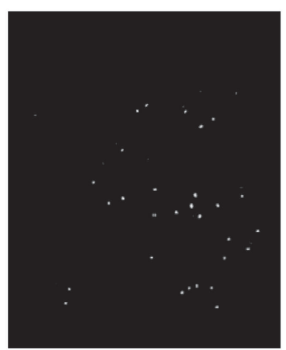

(f)

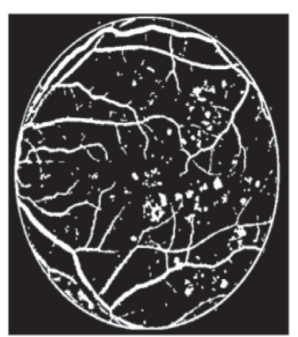

(c)

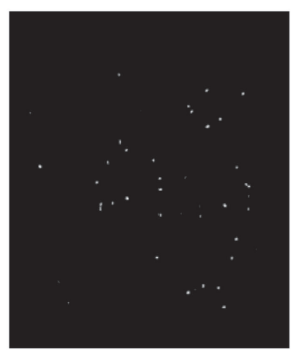

(g)

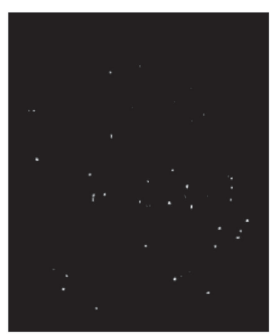

(d)

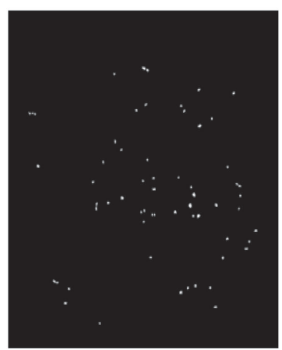

(h)

Figure 4. Retinal image (a) original RGB image, (b) Gray image from Green channel, (c) Image after optic disk removal and vessel detection, (d) Local Relative Entropy-Based Thresholding, (e) Inverse Adaptive Surface Thresholding, (f) Inverse Segmentation Method, (g) Dark Object Segmentation, (h) Proposed Algorithm. 
matching. Multi-Layer perceptron (MLP) Network is used for classification of red lesions. The performance of the proposed algorithm is better compared with the existing algorithms.

MLP is a feedforward neural network with one or more layers between the input and output layers. This type of network is trained with the backpropagation learning algorithm. MLPs are widely used for pattern classification, recognition, prediction and approximation. Multi-Layer Perceptron can solve problems which are not linearly separable. The backpropagation algorithm trains a given feed-forward multilayer neural network for a given set of input patterns with known classifications. When each entry of the sample set is presented to the network, the network examines its output response to the sample input pattern. The output response is then compared to the known and desired output and the error value is calculated. Based on the error, the connection weights are adjusted. When a Back Propagation network is cycled, the activations of the input units are propagated forward to the output layer through the connecting weights. The net input to a unit is determined by the weighted sum of its inputs:

$$
n e t_{j}=\sum_{i} w_{j i} a_{i}
$$

where $a_{i}$ is the input activation from unit $i$ and $w_{j i}$ is the weight connecting unit $i$ to unit $j$. The net input is added to the unit's bias $\theta$ and the resulting value is passed through a sigmoid function:

$$
F\left(\text { net }_{j}\right)=\frac{1}{\left(1+e^{-n e t_{j}}+\theta_{j}\right)}
$$

The bias term plays the same role as the threshold in the perceptron. The output of a sigmoid is a continuous real-value between 0 and 1 . The sigmoid function maps its inputs onto a fixed range. Learning in a back propagation network is in two steps. First, each input pattern $p$ is presented to the network and propagated forward to the output. Second, a method called gradient descent is used to minimize the total error on the patterns in the training set. In gradient descent, weights are changed in proportion to the negative of an error derivative with respect to each weight:

$$
\Delta w_{j i}=-\left[\frac{\delta E}{\delta w_{j i}}\right]
$$

Weights move in the direction of steepest descent on the error surface defined by the total error (summed across patterns):

$$
E=\frac{1}{2} \sum_{p} \sum_{j}\left(t_{p j}-o_{p j}\right)^{2}
$$

where $O_{p j}$ is the activation of output unit $u_{j}$ in response to pattern $p$ and $t_{p j}$ is the target output value for unit $u_{j}$.

Confusion matrix is a useful tool for analysing how well the classifier can recognize tuples of different classes. True Positives refer to the positive tuples that were correctly labelled by the classifier. True negatives are the negative tuples that were correctly labelled by the classifier. False positives are the negative tuples that were incorrectly labelled. False negatives are the positive tuples that were incorrectly labelled. Sensitivity is also referred to as true positive rate or recognition rate. It can be used to access the percentage of positive tuple that are correctly 
classified. Specificity is the true negative rate that is the proportion of negative tuples that are correctly identified. Accuracy is the function of sensitivity and specificity. The classification accuracy of the diagnosis is assessed using the sensitivity and specificity measures. The higher the sensitivity and specificity values, the better the diagnosis. Tables 1 and 2 have shown the information about datasets with normal and abnormal images. Tables 3, 4, and 5 have shown

Table 1. Datasets.

\begin{tabular}{lccc}
\hline Databases & No. of images & Training set & Testing set \\
\hline DIARETDB0 & 130 & 80 & 50 \\
DIARETDB1 & 89 & 55 & 34
\end{tabular}

Table 2. Normal and abnormal images.

\begin{tabular}{lccc}
\hline Databases & No. of images & Normal & Abnormal \\
\hline DIARETDB0 & 130 & 20 & 110 \\
DIARETDB1 & 89 & 5 & 84 \\
\hline
\end{tabular}

Table 3. Sensitivity.

\begin{tabular}{lcc}
\hline Algorithms & DIARETDB0 & DIARETDB1 \\
\hline TMWD & 98.32 & 98.91 \\
DOS & 96.89 & 97.5 \\
IS & 97.4 & 98.34 \\
IAST & 98.91 & 98.7 \\
LRET & 99.85 & 100 \\
\hline
\end{tabular}

TMWD, Template matching in wavelet domain; DOS, Dark object Segmentation; IS, Inverse segmentation; IAST, Inverse adaptive surface thresholding; LRET, Local relative entropybased thresholding.

Table 4. Specificity.

\begin{tabular}{lcc}
\hline Algorithms & DIARETDB0 & DIARETDB1 \\
\hline TMWD & 97.59 & 97.65 \\
DOS & 95.22 & 96.28 \\
IS & 96.8 & 97.09 \\
IAST & 98.62 & 97.4 \\
LRET & 95.78 & 94.14 \\
\hline
\end{tabular}

Table 5. Accuracy.

\begin{tabular}{lcc}
\hline Algorithms & DIARETDB0 & DIARETDB1 \\
\hline TMWD & 97.86 & 98.33 \\
DOS & 96.05 & 96.89 \\
IS & 97.1 & 97.71 \\
IAST & 98.76 & 98.1 \\
LRET & 97.82 & 97.1 \\
\hline
\end{tabular}


the performance comparison between the proposed approach and existing algorithms in terms of sensitivity, specificity and accuracy.

$$
\begin{aligned}
& \text { Sensitivity }=T P /(T P+F N) \\
& \text { Specificity }=T N /(T N+F P) \\
& \text { Precision }=T P /(T P+T N)
\end{aligned}
$$

where

$T P$ is the number of exudates that are correctly identified.

$T N$ is the number of non-exudates that are correctly identified.

$F P$ is the number of non-exudates found as exudates.

$F N$ is the number of exudates found as non-exudates.

Accuracy $=$ sensitivity (no. of positive tuples/no. of positive tuples + no. of negative tuples) *specificity (no. of negative tuples /no. of positive tuples + no. of negative tuples).

\section{Conclusion}

Wavelet-based template matching algorithm was developed to detect microaneurysms. Wavelets were used to automate the multi-scale Gaussian kernels using MCMC method. The base map was created using different orthogonal transforms. From the base map, texton base map was created. The texton-based method was invariant to changes in illumination and orientation. Wavelet decomposition will proceed based on the minimum description length principle. Gaussian mixture model and microstructure feature extraction techniques were used for the segmentation of abnormal retinal lesions. The proposed algorithm achieved better segmentation results than conventional segmentation algorithms. Figures 5 and 6 have shown the performance analysis using DIARETDB0 and DIARETDB1 datasets respectively.

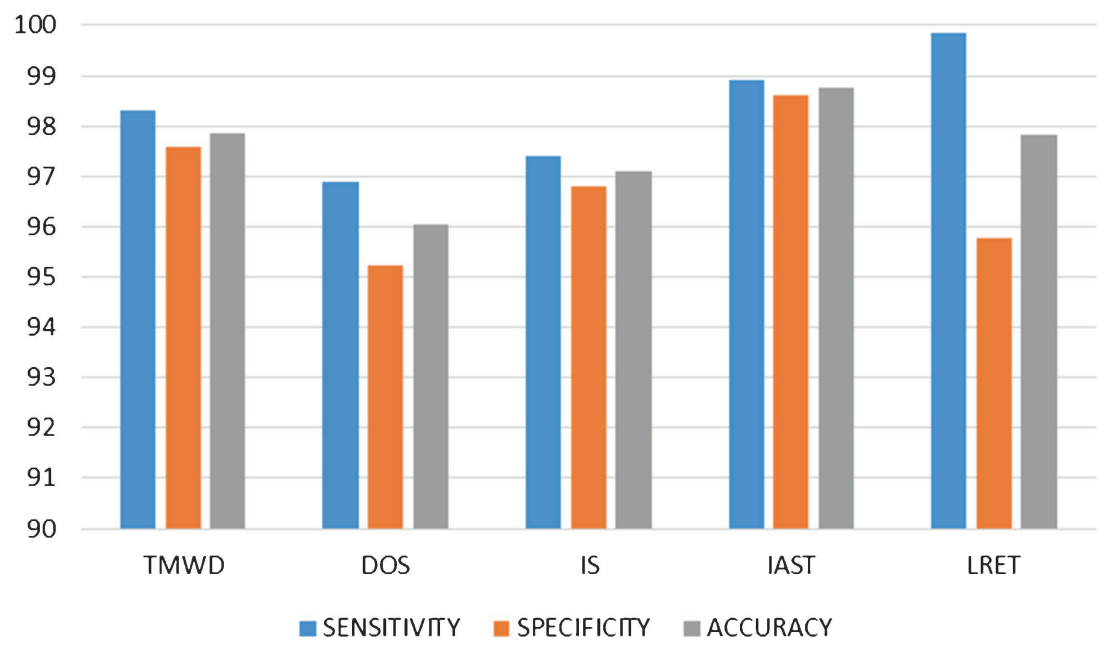

Figure 5. Performance analysis using DIARETDB0 dataset. 


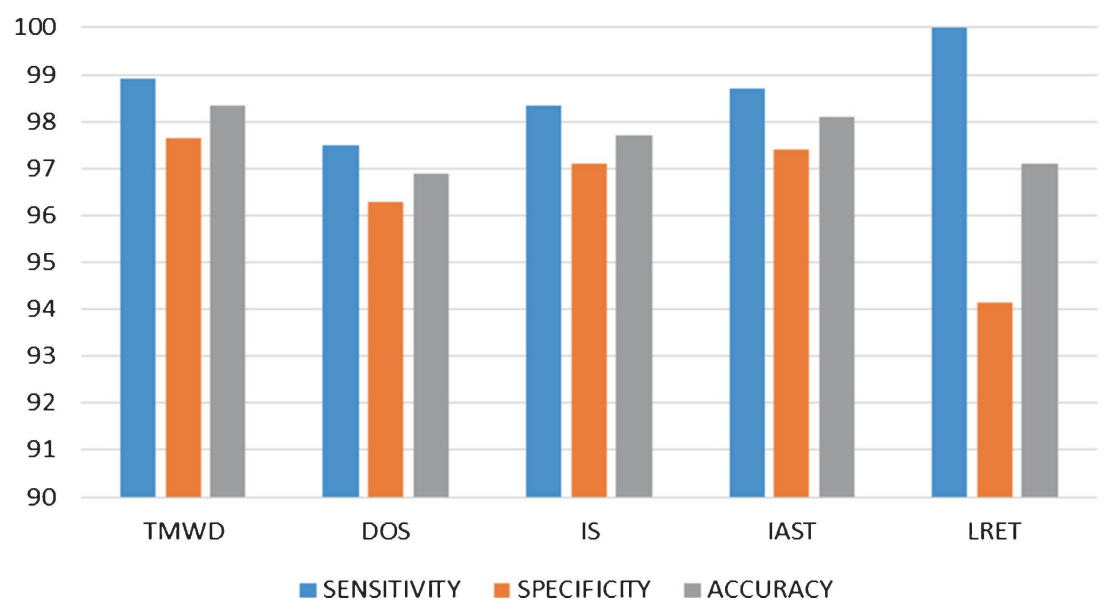

Figure 6. Performance analysis using DIARETDB1 dataset.

\section{References}

Alan D Fleming, Sam Philip, Keith A Goatman, John A Olson and Peter F Sharp 2006 Automated microaneurysm detection using local contrast normalization and local vessel detection. IEEE Trans. Med. Imaging 25(9): 1223-1232

Anderson Rocha, Tiago Carvalho, Herbert F Jelinek, Siome Goldenstein and Jacques Wainer 2012 Points of interest and visual dictionaries for automatic retinal lesion detection. IEEE Trans. Biomed. Eng. 59(8): 2244-2253

Balint Antal and Andras Hajdu 2012 An ensemble-based system for microaneurysm detection and diabetic retinopathy grading. IEEE Trans. Biomed. Eng. 59(6): 1720-1726

Balint Antal, Andras Hajdu, Zsuzsanna Maros-Szabo, Zsolt Torok, Adrienne Csutak and Tunde Peto 2012 A two-phase decision support framework for the automatic screening of digital fundus images. $J$. Comput. Sci. 3(5): 262-268

Bob Zhang, Fakhri Karray, Qin Li and Lei Zhang 2012 Sparse representation classifier for microaneurysm detection and retinal blood vessel extraction. Inf. Sci. 200: 78-90

Bob Zhang, Xiangqian Wu, Jane You, Qin Li and Fakhri Karray 2010 Detection of microaneurysms using multi-scale correlation coefficients. Pattern Recogn. 43(6): 2237-2248

Carla Agurto, Victor Murray, Eduardo Barriga, Sergio Murillo, Marios Pattichis, Herbert Davis, Stephen Russell, Michael Abràmoff and Peter Soliz 2010 Multiscale AM-FM methods for diabetic retinopathy lesion detection. IEEE Trans. Med. Imaging 29(3): 502-512

Cemal Kose, Ugur Sevik, Cevat Ikibas and Hidayet Erdol 2012 Simple methods for segmentation and measurement of diabetic retinopathy lesions in retinal fundus images. Comput. Methods Programs Biomed. 107(2): 274-293

Fangyan Nie, Yonglin Wang, Meisen Pan, Guanghan Peng and Pingfeng Zhang 2013 Two-dimensional extension of variance-based thresholding for image segmentation. Multidim. Syst. Sign. Process. 24(3): 485-501

Giri Babu Kande, T Satya Savithri and P Venkata Subbaiah 2010 Automatic detection of microaneurysms and hemorrhages in digital fundus images. J. Digit. Imaging 23(4): 430-437

Istvan Lazar and Andras Hajdu 2013 Retinal microaneurysm detection through local rotating cross-section profile analysis. IEEE Trans. Med. Imaging 32(2): 400-407

Marwan D Saleh and Eswaran C 2012 An automated decision-support system for non-proliferative diabetic retinopathy disease based on MAs and HAs detection. Comput. Methods Programs Biomed. 108(1): $186-196$ 
Meindert Niemeijer, Bram van Ginneken, Joes Staal, Maria S A Suttorp-Schulten and Michael D Abràmoff 2005 Automatic detection of red lesions in digital color fundus photographs. IEEE Trans. Med. Imaging 24(5): 584-592

Meysam Tavakoli, Reza Pourreza Shahri, Hamidreza Pourreza, Alireza Mehdizadeh, Touka Banaee and Mohammad Hosein Bahreini Toosi 2013 A complementary method for automated detection of microaneurysms in fluorescein angiography fundus images to assess diabetic retinopathy. Pattern Recognit. 46(10): 2740-2753

Mohammed Al-Rawi, Munib Qutaishat and Mohammed Arrar 2007 An improved matched filter for blood vessel detection of digital retinal images. Comput. Biol. Med. 37(2): 262-267

Thomas Walter, Pascale Massin, Ali Erginay, Richard Ordonez, Clotilde Jeulin and Jean-Claude Klein 2007 Automatic detection of microaneurysms in color fundus images. Med. Image Anal. 11(6): 555-566

Usman Akram M and Shoab A Khan 2013 Multi-layered thresholding-based blood vessel segmentation for screening of diabetic retinopathy. Eng. Comput. 29(2): 165-173 